\title{
Fabrication and Application of Iron(III)-Oxide Nanoparticle/Polydimethylsiloxane Composite Cone in Microfluidic Channels
}

\author{
Cheng-Chun Huang, ${ }^{1}$ Ming-Dao Wu, ${ }^{1}$ Dao Liang, ${ }^{1}$ Jiashing Yu, ${ }^{2}$ \\ Po-Jen Shih, ${ }^{3}$ and Wen-Pin Shih ${ }^{1}$ \\ ${ }^{1}$ Department of Mechanical Engineering, National Taiwan University, Taipei 10617, Taiwan \\ ${ }^{2}$ Department of Chemical Engineering, National Taiwan University, Taipei 10617, Taiwan \\ ${ }^{3}$ Department of Civil and Environmental Engineering, National University of Kaohsiung, Kaohsiung 81148, Taiwan
}

Correspondence should be addressed to Wen-Pin Shih, wpshih@ntu.edu.tw

Received 4 May 2012; Accepted 5 December 2012

Academic Editor: Gaurav Mago

Copyright (c) 2012 Cheng-Chun Huang et al. This is an open access article distributed under the Creative Commons Attribution License, which permits unrestricted use, distribution, and reproduction in any medium, provided the original work is properly cited.

This paper presented the fabrication and applications of an iron(III)-oxide nanoparticle/polydimethylsiloxane (PDMS) cone as a component integrated in lab on a chip. The two main functions of this component were to capture magnetic microbeads in the microfluid and to mix two laminar fluids by generating disturbance. The iron(III)-oxide nanoparticle/PDMS cone was fabricated by automatic dispensing and magnetic shaping. Three consecutive cones of $300 \mu \mathrm{m}$ in height were asymmetrically placed along a microchannel of $2 \mathrm{~mm}$ in width and $1.1 \mathrm{~mm}$ in height. Flow passing the cones was effectively redistributed for Renolds number lower than $3.45 \times 10^{-3}$. Streptavidin-coated magnetic microbeads which were bound with biotin were successfully captured by the composite cones as inspected under fluorescence microscope. The process parameters for fabricating the composite cones were investigated. The fabricated cone in the microchannel could be applied in lab on a chip for bioassay in the future.

\section{Introduction}

Microfluidic device applied in chemical and biological process has drawn more and more attention in research due to versatile advantages such as low fabrication cost, short reaction time, and low reagent consumption. However, the laminar characteristics of flow in microchannel make it difficult to mix two separate flow streams together. Many mixing methodologies have been reported to enhance the mixing efficiency. One of the popular mixing methods is to generate chaotic advection utilizing particular geometric structures in microchannel, such as staggered herringbone pattern on channel surface $[1,2]$, triangular-wave structures as obstacles along channel side walls [3], and spiral microchannels [4]. Furthermore, PDMS multilayer microfluidic chips with 3D configuration have been fabricated simply to carry out mixing work [5].

Magnetic beads are popular carriers for biological manipulation in microfluidic system under the application of magnetic field. Particularly, bioanalytic processes such as separation, capture, and recognition [6-9] could be conducted and integrated utilizing magnetic beads. In previous studies [10-13], the magnetic fields were applied from the outside of device with permanent magnet. If the magnetic fields could be generated in specified locations in a microchannel, the external magnet would not be needed and hence the device implementation would be made easier.

In this paper, we fabricate a magnetic nanoparticle/polydimethylsiloxane (PDMS) microcone for mixing fluids due to its three-dimensional asymmetric shape in a microfluidic chip. The fabricated microcone could also be used to capture magnetic beads with its intrinsic magnetism.

\section{Design and Experiment}

Figure 1 depicts the design of the microfluidic chip composed of three polymethylmethacrylate (PMMA) layers. The microfluidic chip possesses two inlet holes which allow 


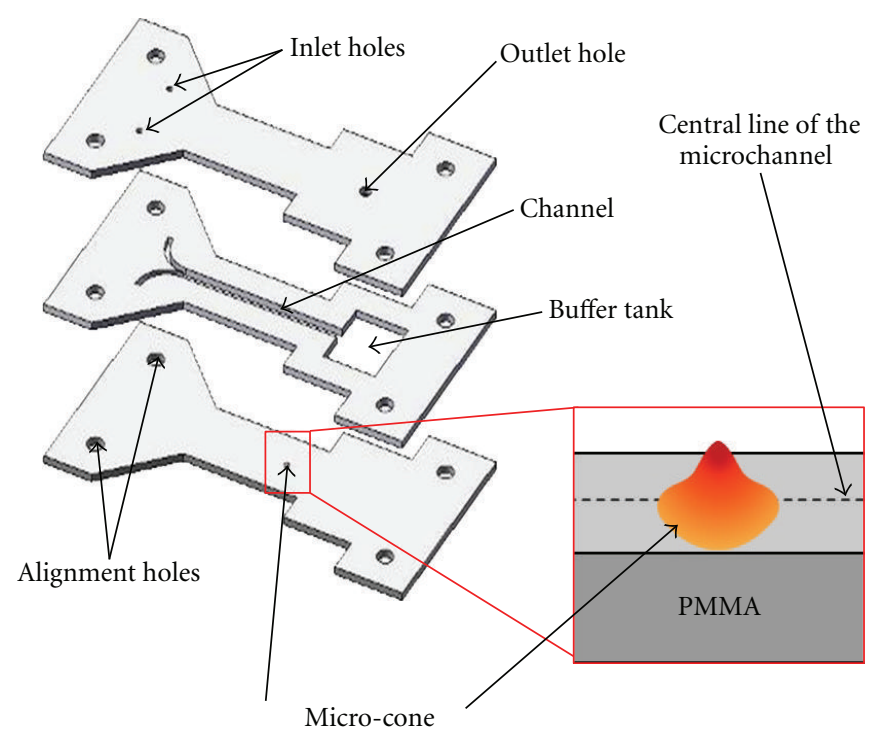

Figure 1: Schematics of the proposed microcone in a microfluidic chip.

two different fluids to enter the microchannel and then to encounter the micro-cone. The fluids can be collected in the buffer tank and then flow out through the outlet hole. There are four alignment holes on each PMMA layer for accurately assembling the microfluidic chip.

Figure 2 illustrates the functionalities of the proposed micro-cone in the microfluidic chip. Let the first fluid in the microchannel contain particles $\mathrm{A}$, which do not possess magnetism, and particles $\mathrm{C}$ attached on magnetic beads. The second fluid contains only particles B. The micro-cone is proposed to separate particles A from particles $C$ and then to mix particles A with particles B together. Before the fluids pass through the micro-cone, they are laminar flows in the microchannel. The particles $\mathrm{C}$ would be captured by the micro-cone due to the magnetic force when the first fluid passes through the micro-cone. Beyond the micro-cone, the first and the second fluids could be mixed due to the turbulence caused by the cone shape. Therefore, particles $\mathrm{A}$ and $\mathrm{B}$ could interact. The particles $\mathrm{C}$ captured on the micro-cone and the mixture of particles $\mathrm{A}$ and $\mathrm{B}$ can then be analyzed for versatile applications of lab on a chip. It should be noted that the position of the proposed micro-cone has to be deviated from the central line of the microchannel for enhancing the mixing effect. In our design, the deviated distance is $10 \%$ of the microchannel width.

\section{Fabrication}

Figure 3 details the process for fabricating the microfluidic chip. It is composed of three PMMA layers. All the layers are machined using a $\mathrm{CO}_{2}$ laser. Each layer is made of a bulk PMMA plate of $1 \mathrm{~mm}$ in thickness. To assemble the microfluidic chip, both sides of the middle layer are coated with adhesive of $50 \mu \mathrm{m}$ in thickness (Figure 3(a)). Therefore, the total depth of the microchannel is $1.1 \mathrm{~mm}$, and the width is $2 \mathrm{~mm}$.

To fabricate the micro-cone, the PDMS prepolymer and curing agent with 10:1 weight ratio are prepared, then

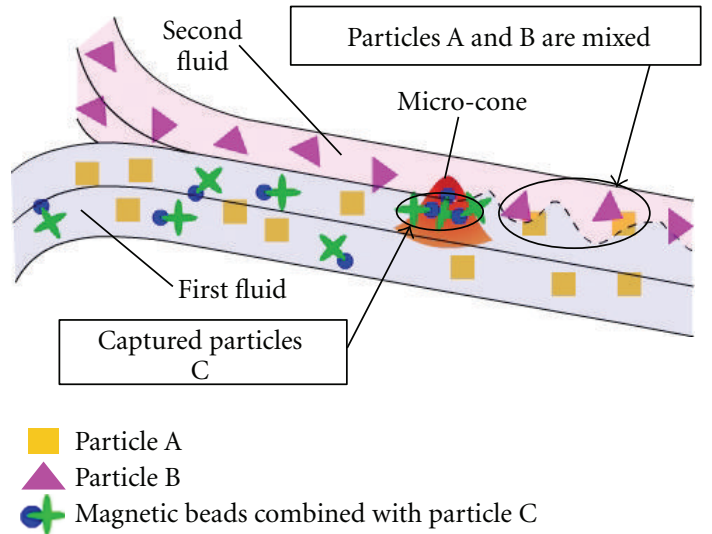

FIGURE 2: Illustrative function of the proposed microcone in a microfluidic chip.

the iron(III)-oxide nanoparticles are dispersed and mixed thoroughly in the PDMS for 10 minutes using regular stirring machine. The mixture is then put in a vacuum chamber for degassing. The iron(III)-oxide nanoparticles (nanopowder of 544884-5G Iron (III)-oxide, SIGMA-ALDRICH) are comprised of primarily the gamma-form $\mathrm{Fe}_{2} \mathrm{O}_{3}$ which exhibits superparamagnetic behavior [14-17]. The average diameter of the nanoparticles is $50 \mathrm{~nm}$. After degassing, the nanoparticle/PDMS composite is poured into a syringe and then degassing process is conducted again. An automatic dispenser is used to extrude the nanoparticle/PDMS composite into the microchannel. In the beginning of the dispensing process, the initial portion of the nanoparticle/PDMS composite is disregarded in order to obtain constant volume of every single drop. A reference plate with a defined area is put onto the sample stage for aligning the dispenser (Figures 4 and 5). Therefore, the syringe needle can be precisely placed above the designated area. After finishing the alignment, the reference plate is replaced by the bottom 


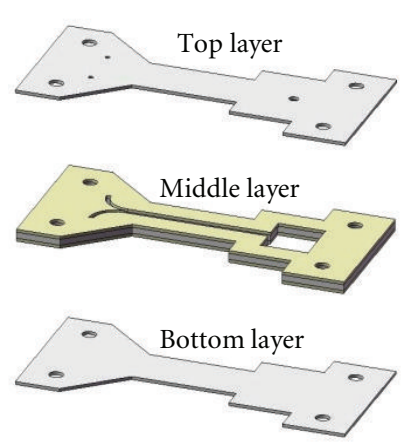

(a)

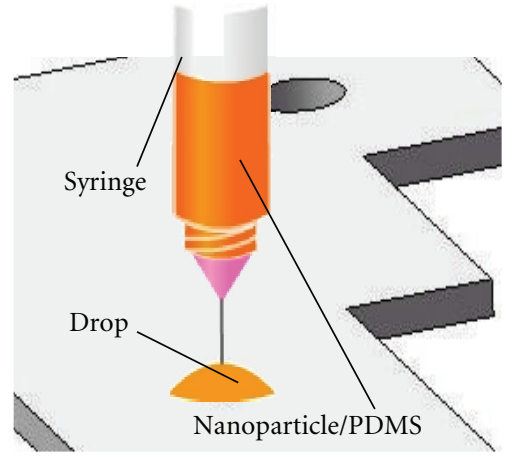

(b)

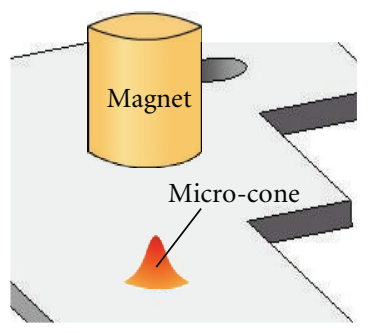

(c)

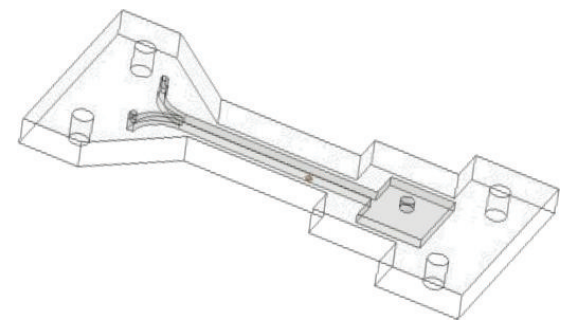

(d)

Figure 3: Process for fabricating the nanoparticle/PDMS micro-cone. (a) The chip is composed of three bulk PMMA plates. (b) The nanoparticle/PDMS composite is applied through the automatic dispenser. (c) The micro-cone is formed under the applied external magnetic field. (d) Schematic of the fabricated microfluidic chip.

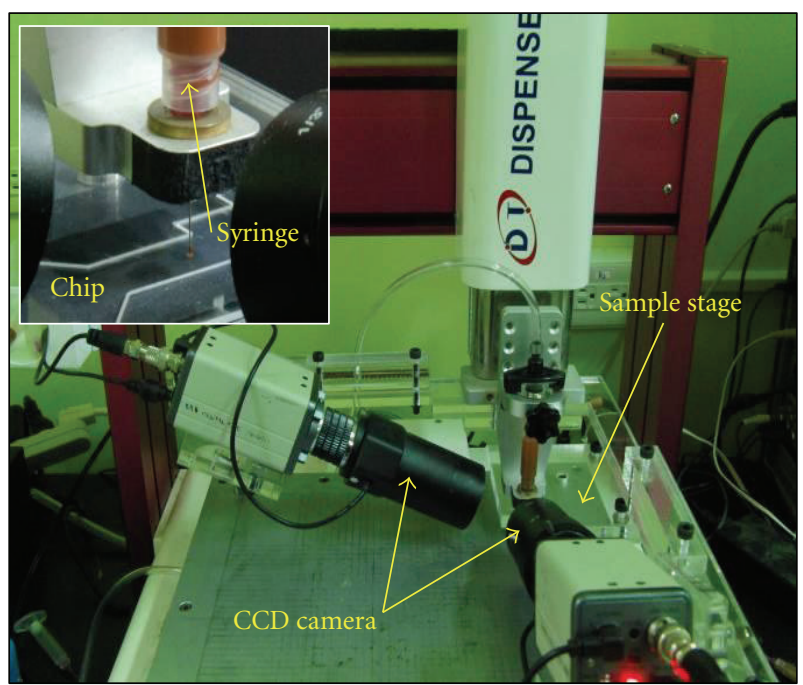

FIgURE 4: Configuration of the automatic dispensing system.

layer of the microfluidic chip. Then a drop of nanoparticle/PDMS composite is dispensed onto the bottom layer (see Figure 3(b)). The dispensing pressure is $0.25 \mathrm{MPa}$. After dispensing the nanoparticle/PDMS composite, a permanent magnet is placed above the composite (Figure 3(c)). The composite becomes a cone shape due to the external magnetic field. Then the cone is cured at $70^{\circ} \mathrm{C}$ for 30 minutes. The fabrication environment is at $23^{\circ} \mathrm{C}$ and $71 \%$ relative humidity. After the cone is cured, the three layers of the microfluidic chip are assembled together (Figure $3(\mathrm{~d})$ ).

There are two setups to facilitate the fabrication process. One is the automatic dispensing system (Figure 4), and

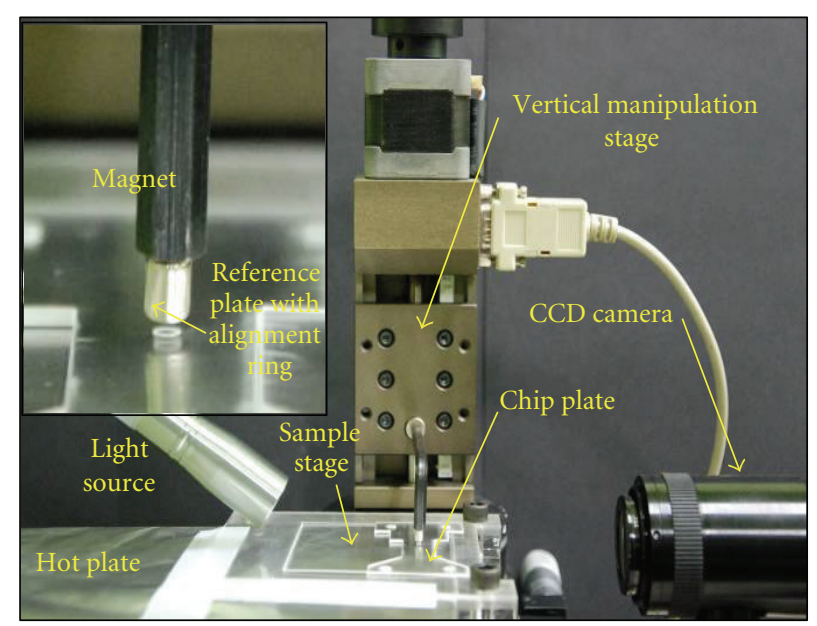

FIgURE 5: Configuration of the magnetic platform.

the other is the magnetic platform for generating the cone shape of the composite (Figure 5). The automatic dispensing system consists of a dispenser (SR-330D), two CCD cameras, and a sample stage. The dispenser is used to extrude iron(III)-oxide nanoparticle/PDMS composites. It features three-axis movement with $50 \mu \mathrm{m}$ resolution and a controller for adjusting the dispensing pressure and duration. The two CCD cameras assist the alignment of the syringe needle on the substrate and monitor the dispensing process. The sample stage is used to displace the substrate. The magnetic platform includes a vertical manipulation stage, a permanent magnet, a CCD camera, a light source, a sample stage, and a hot plate. The permanent magnet is connected to 


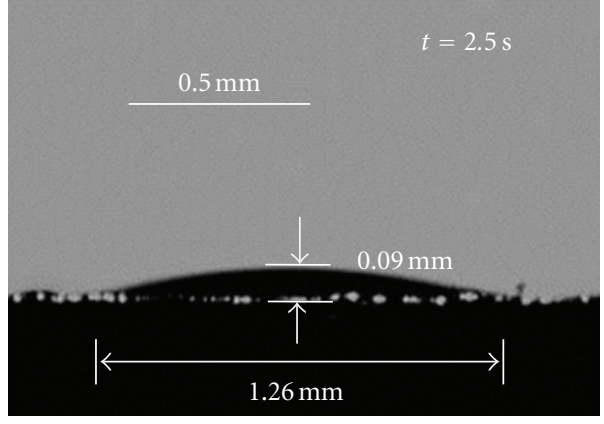

(a)

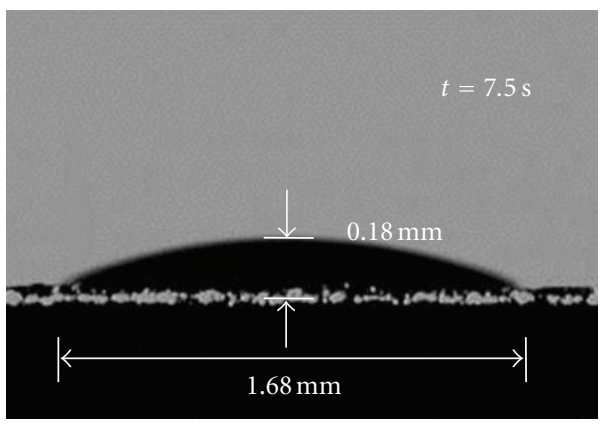

(c)

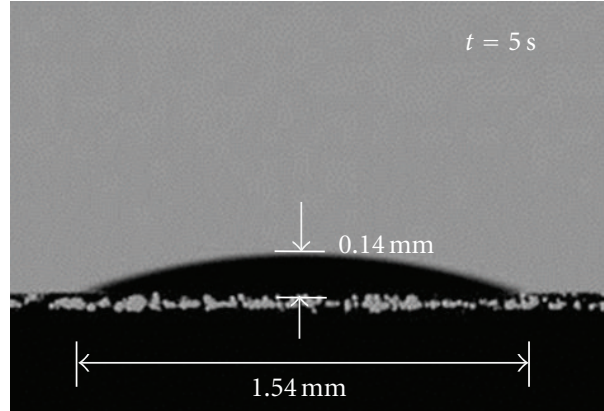

(b)

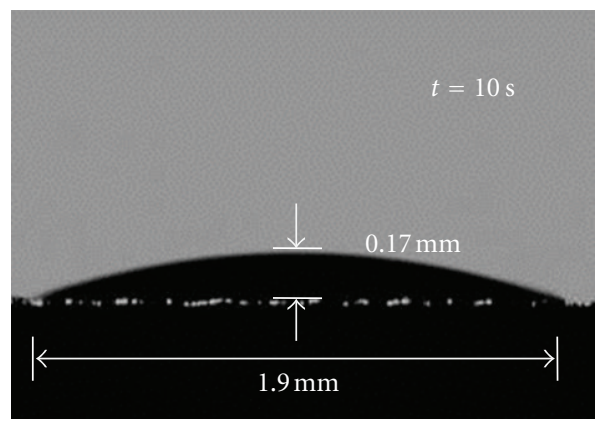

(d)

Figure 6: Images of the PDMS droplets at different dispensing durations $(t)$. The droplet height $(h)$ and diameter $(d)$ are measured. (a) $t=2.5 \mathrm{~s}$. (b) $t=5 \mathrm{~s}$. (c) $t=7.5 \mathrm{~s}$. (d) $t=10 \mathrm{~s}$.

the vertical manipulation stage with $10 \mathrm{~nm}$ resolution. The high resolution of the manipulation stage is necessary to finely adjust the distance between the bottom of the magnet and surface of the substrate. The CCD camera is used to monitor the deformation of the uncured nanoparticle/PDMS composite. There is also a sample alignment stages which is placed on the hot plate so that the alignment would not be deviated in the curing process.

The composites with different weight ratios of the nanoparticles are added into the PDMS for evaluating the fabrication parameters. The weight ratios are prepolymer: curing agent: iron(III)-oxide nanoparticle $=40: 4: 1$, $40: 4: 3,40: 4: 5$, respectively. Therefore, the weight ratios of the nanoparticles to the composite are $2.22 \%, 6.38 \%$, and $10.20 \%$, respectively. Different magnetic fields which are controlled by the distance $(D)$ from the bottom of the magnet to the substrate surface are applied. The corresponding magnetic fields of variant distances between substrate and magnet are measured by tesla meter (TM-701, KANETEC CO., LTD). The results are summarized in Table 1.

\section{Results}

The size of drops could be controlled by the diameter of syringe needle, dispensing pressure and dispensing duration. The stainless steel syringe needle which its inner diameter is $0.15 \mathrm{~mm}$ and outer diameter is $0.3 \mathrm{~mm}$ was used. The dispensing pressure is fixed at $0.25 \mathrm{MPa}$. Figure 6 shows the images of PDMS droplets without nanoparticles at different dispensing durations $t=2.5,5,7.5$, and $10 \mathrm{~s}$. The variation of the droplet size at the same dispensing
TABle 1: Weight ratios of nanoparticles and magnet height for parametric study.

\begin{tabular}{lccc}
\hline & \multicolumn{3}{c}{ Weight ratio of nanoparticles } \\
& $2.22 \%$ & $6.38 \%$ & $10.20 \%$ \\
\hline & $1.0 \mathrm{~mm}$ & $1.25 \mathrm{~mm}$ & $1.5 \mathrm{~mm}$ \\
Distances between & $\mathbf{0 . 2 2 0 ~ T}$ & $\mathbf{0 . 1 9 1 ~ T}$ & $\mathbf{0 . 1 6 8 ~ T}$ \\
substrate and magnet & $1.25 \mathrm{~mm}$ & $1.5 \mathrm{~mm}$ & $1.75 \mathrm{~mm}$ \\
$(\mathrm{~mm})$ & $\mathbf{0 . 1 9 1 ~ T}$ & $\mathbf{0 . 1 6 8 ~ T}$ & $\mathbf{0 . 1 4 8 ~ T}$ \\
Magnetic field (Tesla, T) & $1.5 \mathrm{~mm}$ & $1.75 \mathrm{~mm}$ & $2.0 \mathrm{~mm}$ \\
& $\mathbf{0 . 1 6 8} \mathbf{T}$ & $\mathbf{0 . 1 4 8} \mathbf{T}$ & $\mathbf{0 . 1 3 3} \mathbf{T}$ \\
\hline
\end{tabular}

parameters is not obvious before and after the nanoparticles adding into the PDMS. This parametric study is conducted for obtaining the desirable droplet diameter $(d)$ and height (h) by controlling the dispensing duration. Since the height of the microchannel is $1.1 \mathrm{~mm}$, the initial height of the PDMS droplet should be relatively smaller. All the initial heights of the PDMS droplets using different dispensing durations in our tests are smaller than $1.1 \mathrm{~mm}$, as shown in Figure 7(a). The average height is $h=0.09( \pm 0.007), 0.14( \pm 0.005)$, 0.17 ( \pm 0.009$), 0.19( \pm 0.018) \mathrm{mm}$ for $t=2.5,5,7.5,10 \mathrm{~s}$, respectively. Since, in this design, the channel width is $2 \mathrm{~mm}$ and the deviated distance from the cone center to the central line of the microchannel is $0.2 \mathrm{~mm}$, the diameter of the PDMS droplet should be smaller than $1.6 \mathrm{~mm}$. The obtained cone diameter is shown in Figure 7(b). The average diameter is $d=1.28( \pm 0.029), 1.51( \pm 0.032), 1.64( \pm 0.061)$, and $1.79( \pm 0.067) \mathrm{mm}$ for $t=2.5,5,7.5$, and $10 \mathrm{~s}$, respectively. Therefore, the optimum dispensing duration is $5 \mathrm{~s}$ in this test. 


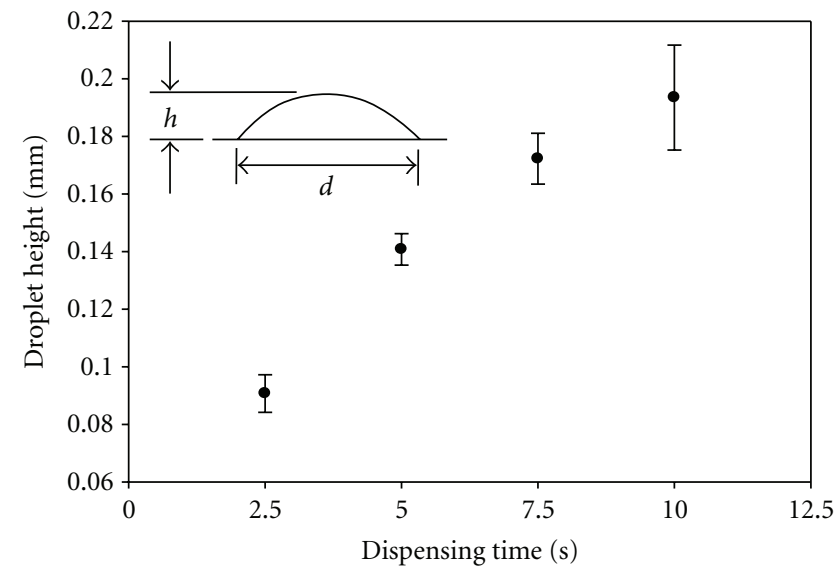

(a)

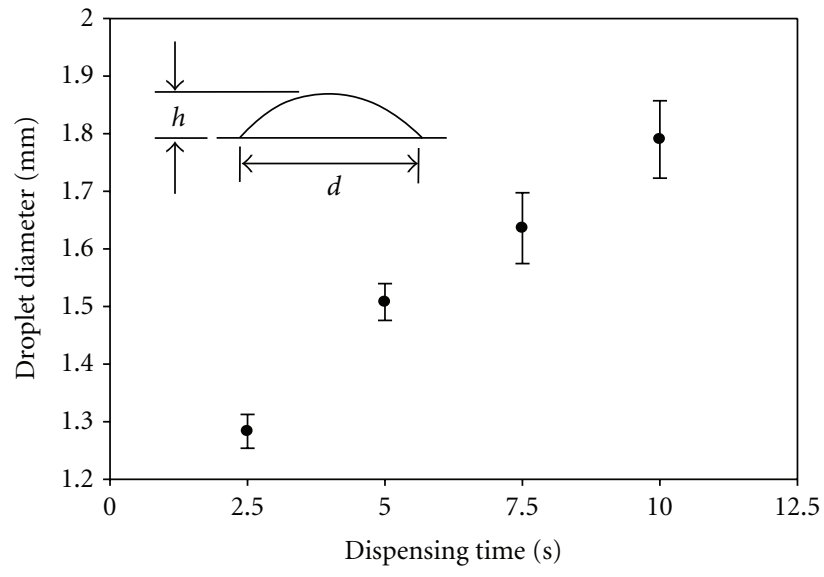

(b)

FIGURE 7: Dispensing test for controlling the droplet height and diameter at the constant dispensing pressure of $0.25 \mathrm{MPa}$. (a) The relation between the droplet height and dispensing duration. (b) The relation between the droplet diameter and dispensing duration.

For each parameter in Table 1, the images of the cone formation are captured every $5 \mathrm{~s}$ in $60 \mathrm{~s}$. For the $2.22 \%$ weight ratio with $1.0 \mathrm{~mm}$ magnetic height, the $6.38 \%$ weight ratio with $1.25 \mathrm{~mm}$ magnetic height, and the $10.2 \%$ weight ratio with $1.5 \mathrm{~mm}$ magnetic height, the nanoparticle/PDMS droplets are pulled up and touch the magnet immediately. Therefore, the images of the cone formation for these three particular samples are captured every $1 \mathrm{~s}$. Figure $8(\mathrm{a})$ shows the height increase $(\Delta h)$ of the droplet deformation as a function of process time. All the data in Figure 8(a) are from the samples of the $10.20 \%$ nanoparticles with the magnetic height of $1.5 \mathrm{~mm}, 1.75 \mathrm{~mm}$, and $2.0 \mathrm{~mm}$, respectively. For the same process time, the height increase is larger for the lower magnetic height. The error bars in Figure 8(a) stand for the standard deviation from five samples. The deviations are attributed to the vertical and horizontal alignment between the magnet and the center of the droplet. The nondispersed nanoparticles in the uncured PDMS might also affect the repeatability of the cone formation.

The inset of Figure 8(a) shows the height increase of the droplet deformation for the magnetic height of $1.5 \mathrm{~mm}$. The five samples are pulled up and reach the magnet at the process time of $11,12,19,20$, and $23 \mathrm{~s}$, respectively. Because the magnet is very close to the droplet, the magnetic force is strong and sensitive to the magnetic height. The variation of the initial droplet might significantly deviate the process time for the droplet to reach the magnet. The height increase of the droplet with $1.5 \mathrm{~mm}$ magnetic height and the nanoparticle weight ratio of $2.22 \%, 6.38 \%$, and $10.20 \%$, respectively, are shown in Figure 8(b). The height increase is larger for the higher weight ratio of nanoparticles. Table 2 shows the height increase at the process time of $30,40,50$, and $60 \mathrm{~s}$, respectively. The evolution of the height increase with the increasing process time for the samples with $1.5 \mathrm{~mm}$ magnetic height and $2.22 \%$ nanoparticles is close to that with $1.75 \mathrm{~mm}$ magnetic height and $10.2 \%$ nanoparticles.

For the $1.75 \mathrm{~mm}$ magnetic height in Figure $8(\mathrm{a})$ and the $6.83 \%$ nanoparticles in Figure $8(\mathrm{~b})$, the slope of the curve changes in a piecewise manner with the process time. In
TABle 2: Parameters of weight ratio of the nanoparticles and the magnetic field.

\begin{tabular}{|c|c|c|c|c|c|c|}
\hline & \multirow{2}{*}{ wt. $\%$} & \multicolumn{4}{|c|}{ Height increase, $\Delta h$} \\
\hline & & & $30 \mathrm{~s}$ & $40 \mathrm{~s}$ & $50 s$ & $60 s$ \\
\hline \multirow{4}{*}{$\begin{array}{l}\text { Distance between substrate } \\
\text { and magnet }(\mathrm{mm})\end{array}$} & \multirow{2}{*}{1.5} & 2.22 & 0.033 & 0.040 & 0.048 & 0.054 \\
\hline & & 6.38 & 0.086 & 0.102 & 0.123 & 0.145 \\
\hline & \multirow{2}{*}{1.75} & 6.38 & 0.032 & 0.035 & 0.004 & 0.042 \\
\hline & & 10.2 & 0.032 & 0.038 & 0.043 & 0.051 \\
\hline
\end{tabular}

the beginning of applying the magnetic field, the slope is steep for the process time from $0 \mathrm{~s}$ to $5 \mathrm{~s}$ in Figure 8(a), while the slope becomes smaller for the process time of $5 \sim 25 \mathrm{~s}$. Figure 9(a) shows the fabricated microfluidic chip, and Figure 9(b) shows the cross-sectional image of the microchannel. The nanoparticle/PDMS cone is successfully fabricated in the microchannel. The height of the micro-cone is $300 \mu \mathrm{m}$, and the deviated distance from the central line is $0.3 \mathrm{~mm}$.

\section{Discussions}

The method to form the cone shape of the nanoparticle/ PDMS composite is to exploit the uncured PDMS deformation as result of being dragged by the nanoparticles. The iron(III)-oxide nanoparticles can be attracted by the permanent magnet. They are mixed in the PDMS and enveloped by the entangling polymer chains. As the magnetic nanoparticles are attracted by the magnetic field above, they would move upward and carry the PDMS together. Because the distribution of the magnetic is hyperbolic (Figure 10(a)), the center of the nanoparticle/PDMS composite is subjected to a relatively stronger magnetic force. Conversely, the rim of the composite is subjected to a weaker magnetic force. As a result, the nanoparticle/PDMS cone can be obtained.

When the magnetic field is applied, the nanoparticles in the composite start to aggregate and to form radiated lines on the cone surface (Figure 10(b)) [18]. There are more nanoparticles aggregating on the tip of the cone in 


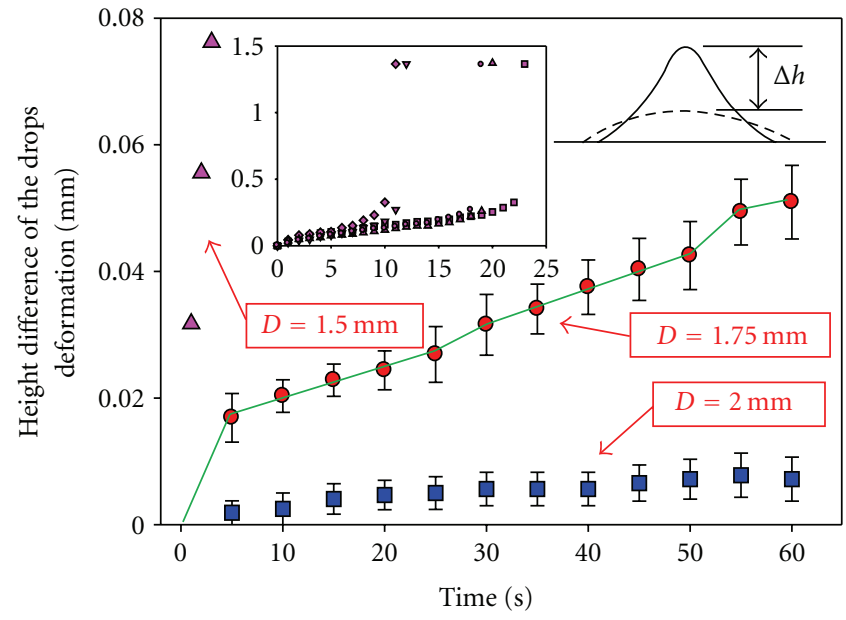

(a)

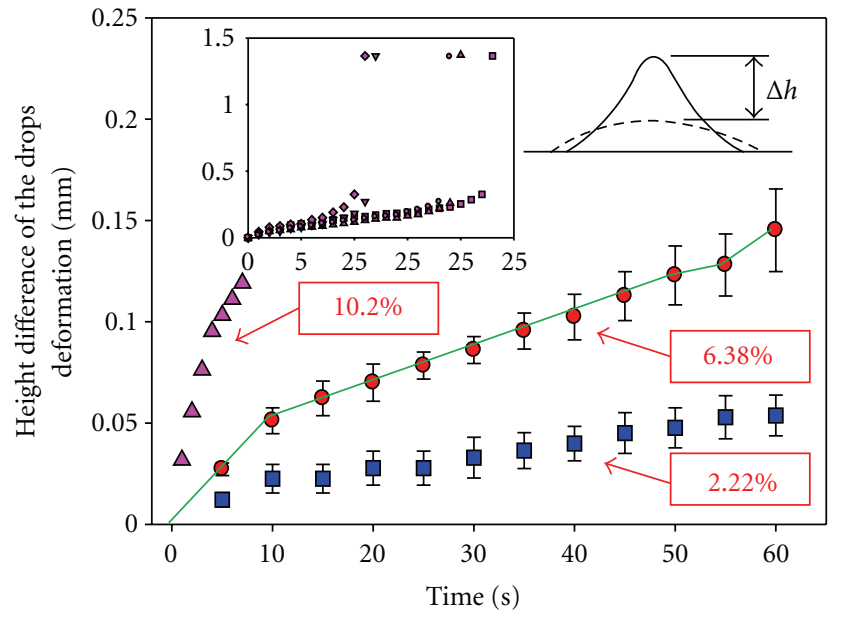

(b)

Figure 8: The height difference, $\Delta h$, of the nanoparticle/PDMS drops deformation. (a) The weight ratio is $10.2 \% . D=1.5,1.75,2.0 \mathrm{~mm}$, respectively. (b) $D=1.5 \mathrm{~mm}$. The weight ratios are $2.22 \%, 6.38 \%$, and $10.2 \%$, respectively.

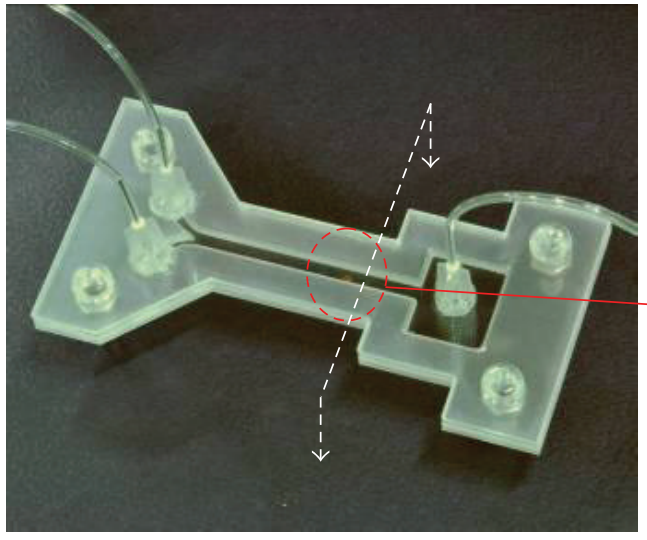

(a)

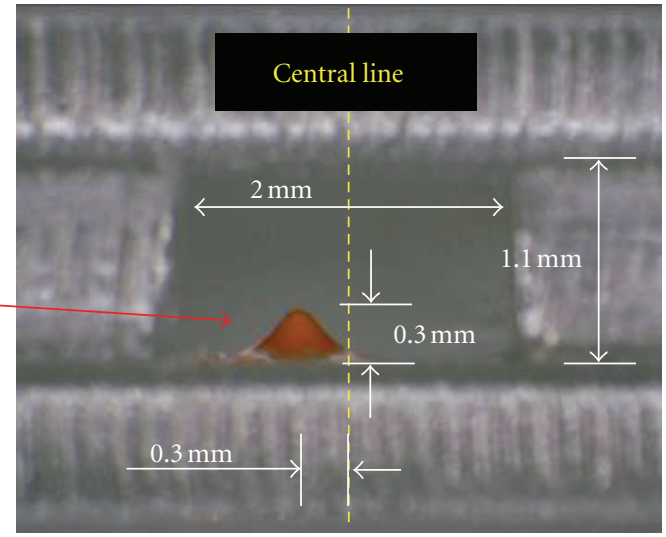

(b)

Figure 9: The images of the fabricated microfluidic chip. (a) The fabricated chip with fluidic interconnects. (b) The cross-sectional view of the nanoparticle/PDMS cone in the microchannel.

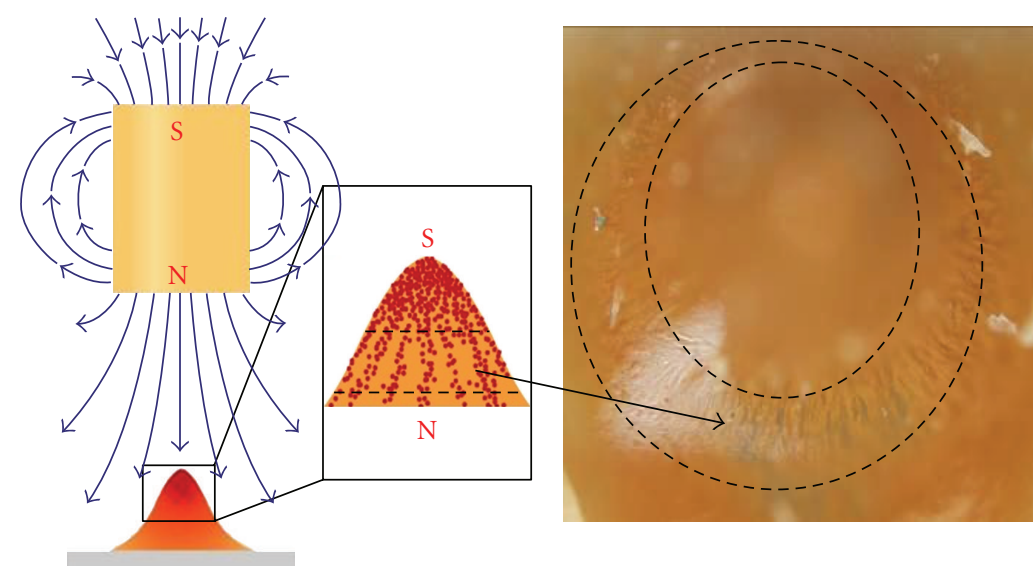

(a)

(b)

Figure 10: Illustration of the magnetism and shape-formation of the cured micro-cone. (a) Schematic of the magnetic field and nanoparticles in PDMS. (b) Top view of the fabricated micro-cone with nanoparticle aggregation. 


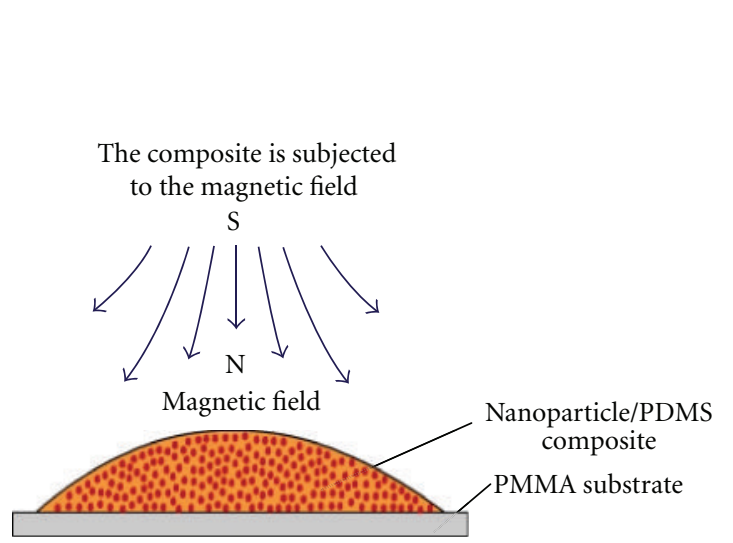

(a)

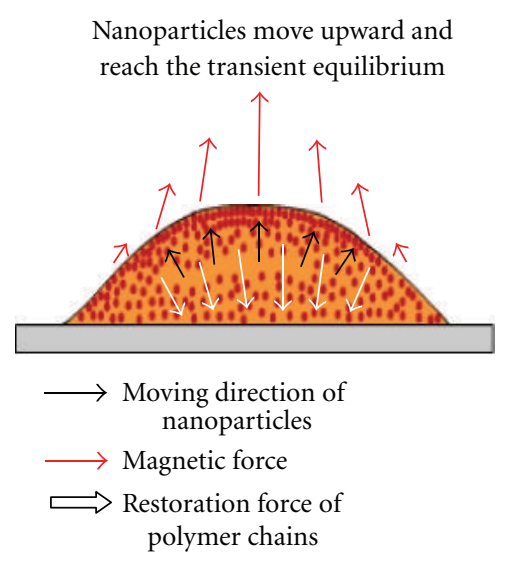

(b)

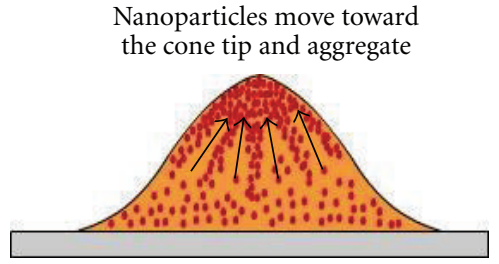

(c)

FIGURE 11: Illustration of the mechanism of the composite deformation.

comparison to the rim region. Therefore, a darker color around the cone tip is observed. Before the nanoparticle/PDMS composite is cured, the nanoparticles are allowed to move in the PDMS. As soon as the PDMS is cured, the nanoparticles cannot move anymore. This results in the gradient magnetism due to the nonuniform distribution of the nanoparticles. Originally, the nanoparticles distribute uniformly in the PDMS. Then the magnetic field on the droplet attracts the nanoparticles which are closer to the droplet surface. These nanoparticles move upward, causing the PDMS to deform as illustrated in Figure 11(a). The momentum of the nanoparticles causes the PDMS to deform rapidly, and hence, the initial slope in Figure 8(a) is steep. When the magnetic force and the restoration force of the polymer chains reach transient equilibrium, the speed of deformation becomes slower, as illustrated in Figure 11(b). Although it is in equilibrium at $5 \mathrm{~s}$ in Figure 8(a), the height increase reduces the distance between the magnet and the top of the droplet. Therefore, the magnetic force exerting on the nanoparticles which are close to the droplet surface increases. Nevertheless, the nanoparticles are insufficient to cause rapid deformation of the PDMS. Hence the slope becomes smaller for the process time during $5 \sim 25 \mathrm{~s}$. Since the top of the droplet is subjected to a relatively stronger magnetic force, the nanoparticles in the internal portion of the droplet will move to the surface gradually and then accumulate on top of the droplet, in the center of the drop top, as illustrated in Figure 11(c). When the nanoparticles are sufficient and the droplet reaches the appropriate height, the droplet deformation becomes more rapid for the process time during 25 30 s in Figure 8(a). The nanoparticles keep moving to the droplet top and hence the PDMS deformation concentrates at the top of the droplet until the cone shape is obtained. The more nanoparticles in the PDMS, the stronger magnetism the cured cone has. However, the shorter magnetic height would cause the cone to touch the magnet. The $6.38 \%$ nanoparticles with the $1.5 \mathrm{~mm}$ magnetic height are the optimum parameters in this study and hence are used in the following fabrication of the microfluidic chip.

Figure 12 shows the deformation process for the $10.2 \%$ nanoparticles at 0,10 , and $20 \mathrm{~s}$ in Figure 8(a). The first
TABLE 3: Reynolds numbers estimation of deionized water and glycerin.

\begin{tabular}{lcc}
\hline & Deionized water & Glycerin \\
\hline$\rho$, density $\left(\mathrm{kg} / \mathrm{m}^{3}\right)$ & 1000 & 1263 \\
$\nu$, velocity $(\mathrm{m} / \mathrm{s})$ & $2.50 \times 10^{-3}$ & $2.50 \times 10^{-3}$ \\
$D_{H}$, hydraulic diameter $(\mathrm{m})$ & $1.05 \times 10^{-3}$ & $1.05 \times 10^{-3}$ \\
$\mu$, dynamic viscosity $(\mathrm{Pa} * \mathrm{~s})$ & $8.90 \times 10^{-4}$ & 0.96 \\
Re, Reynolds numbers & 2.96 & $3.45 \times 10^{-3}$ \\
\hline
\end{tabular}

set of the pictures (Figures 12(a)-12(c)) indicate that the cone for the $1.5 \mathrm{~mm}$ magnetic height is formed quickly at the process time during $20 \mathrm{~s}$ but it is unstable. For the process time beyond $20 \mathrm{~s}$ in Figure 8 (a), the micro-cone will touch the magnet and then break into two parts, as shown in (Figure 12(j)). The smaller portion of the composite is attached to the magnet while the large portion remains on the substrate, as shown in Figure 12(k). The process images for the magnetic height of $1.75 \mathrm{~mm}$ are shown in Figures $12(\mathrm{~d})-12(\mathrm{f})$. The cone is formed gradually but cannot be clearly observed for the process time shorter than $20 \mathrm{~s}$. The process images for the magnetic height of $2.0 \mathrm{~mm}$ are shown in Figures 12(g)-12(i). The composite barely deforms at this magnetic height because the magnetic force is not strong enough to overcome the restoration force of the polymer chains.

\section{Micorchannel Mixing Test}

In order to enhance the mixing efficiency, three nanoparticle/PDMS micro-cones are fabricated in the microchannel. Two kinds of the fluids, deionized water and glycerin, are used for microchannel mixing test. The width of the channel is $2 \mathrm{~mm}$, the height of channel is $1.1 \mathrm{~mm}$, the hydraulic diameter is calculated as $1.05 \mathrm{~mm}$, and the flow rate is set as $10 \mathrm{~mL} / \mathrm{h}$. The Reynolds numbers, $\operatorname{Re}=\rho v D_{H} / \mu$, of these two fluids are listed in Table 3 . In the same condition of the diameters of the channel and the flow rate, however, the Reynolds numbers are different mainly due to variations in the dynamic viscosity, $\mu$. Figures $13(\mathrm{a})$ and $13(\mathrm{~b})$ show 


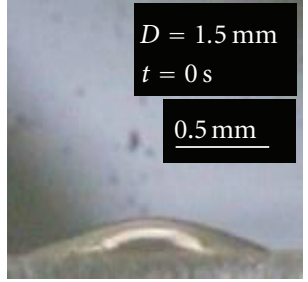

(a)

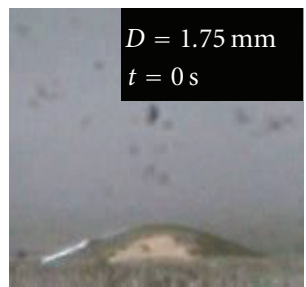

(d)

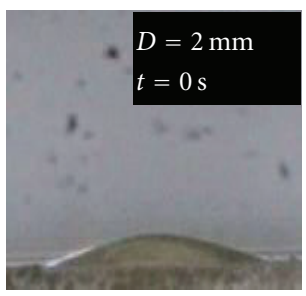

(g)

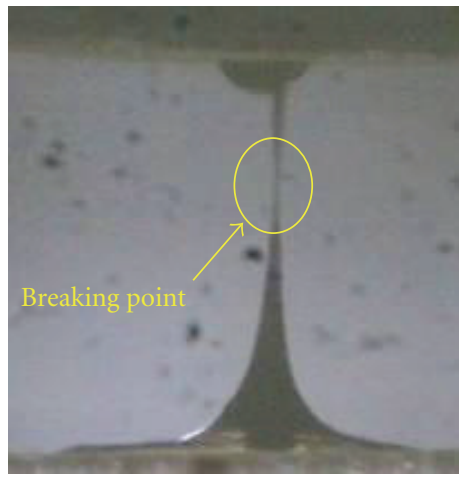

(j)

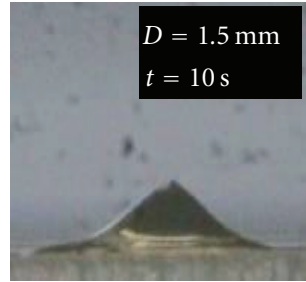

(b)

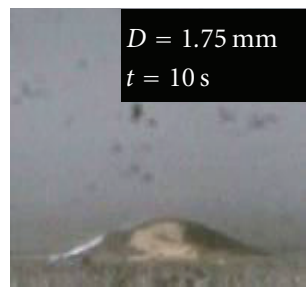

(e)

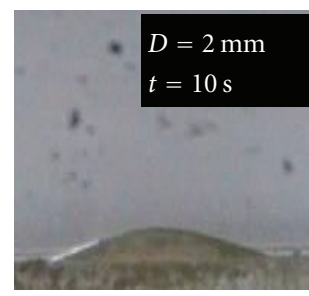

(h)

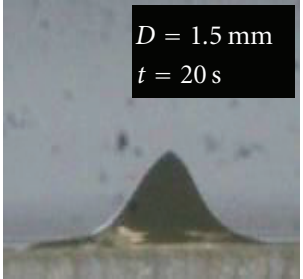

(c)

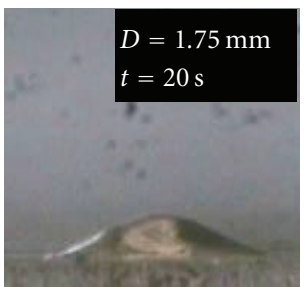

(f)

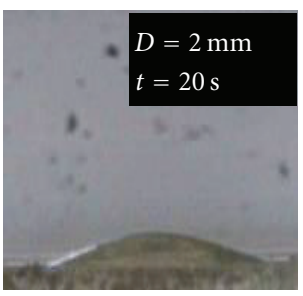

(i)

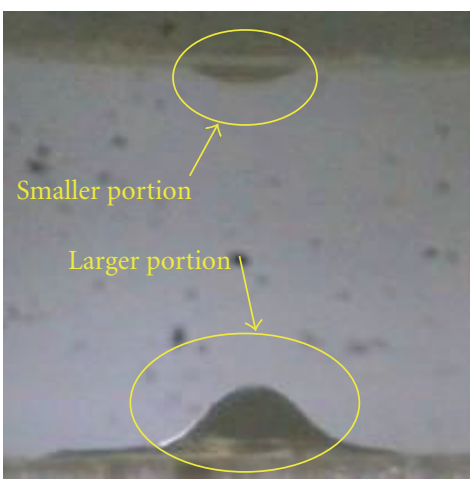

(k)

FIgure 12: The process of the nanoparticle/PDMS droplet becomes a cone shape. The weight ratio of the nanoparticles is $10.2 \%$. (a) $D=$ $1.5 \mathrm{~mm}, t=0 \mathrm{~s}$. (b) $D=1.5 \mathrm{~mm}, t=10 \mathrm{~s}$. (c) $D=1.5 \mathrm{~mm}, t=20 \mathrm{~s}$. (d) $D=1.75 \mathrm{~mm}, t=0 \mathrm{~s}$. (e) $D=1.75 \mathrm{~mm}, t=10 \mathrm{~s}$. (f) $D=1.75 \mathrm{~mm}$, $t=20 \mathrm{~s}$. (g) $D=2.0 \mathrm{~mm}, t=0 \mathrm{~s}$. (h) $D=2.0 \mathrm{~mm}, t=10 \mathrm{~s}$. (i) $D=2.0 \mathrm{~mm}, t=20 \mathrm{~s}$. (j) $D=1.5 \mathrm{~mm}$. The composite which expands between the substrate and the magnetic is about to break. (k) $D=1.5 \mathrm{~mm}$. The composite breaks into two parts.

the fluidic test of deionized water without and with microcone, respectively. Because the Reynolds number is 2.96, which is not low enough, the laminar phenomenon is not obvious. Different diffusion results across the interface between the two fluids are also observed under different Reynolds numbers. In Figure 13(a) without the micro-cone, the distribution of the red-ink water crosses the whole channel width at a relatively downstream location as marked by the line A. In Figure 13(b) with the micro-cones, the distribution of the red-inked water crosses the whole channel width at a relatively upstream location as marked by the line B. The result demonstrates that the micro-cones effectively enhanced the mixing efficiency. Figures 13(c) and 13(d) show the fluidic test of glycerin and the Reynolds number is $3.45 \times 10^{-3}(\operatorname{Re} \ll 1)$. The laminar phenomenon could be seen clearly in Figure 13(c). In Figure 13(d), although the red ink glycerin does not distribute across the channel width through the whole channel length, the flow field is redistributed as passing through the micro-cones and the flow of the red-inked glycerin becomes wider.

\section{Magnetism of the Micro-Cones Test}

Streptavidin has extremely high affinity for biotin, so the streptavidin-coated magnetic particles and fluorescently labeled biotin are combined to carry out the magnetism 


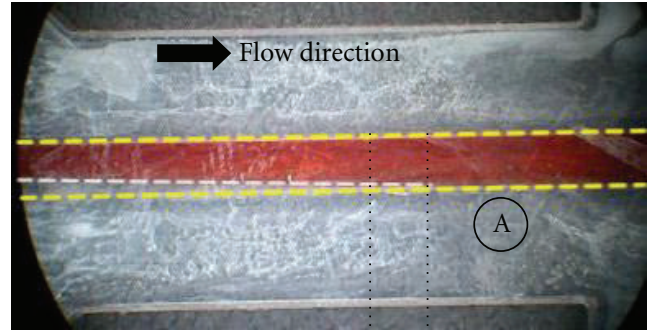

(a)

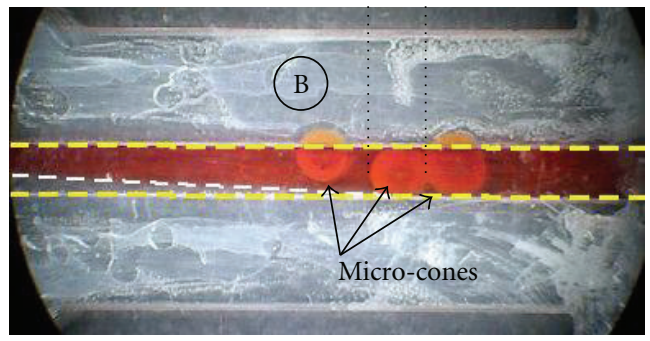

(b)

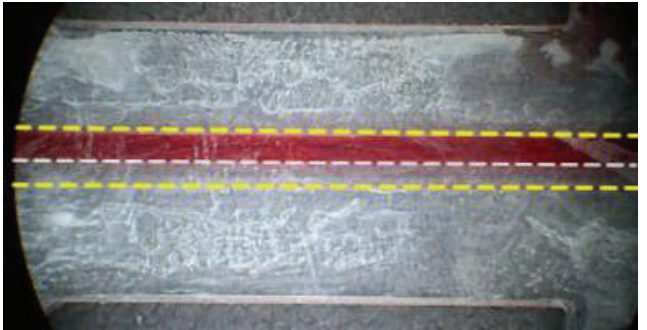

(c)

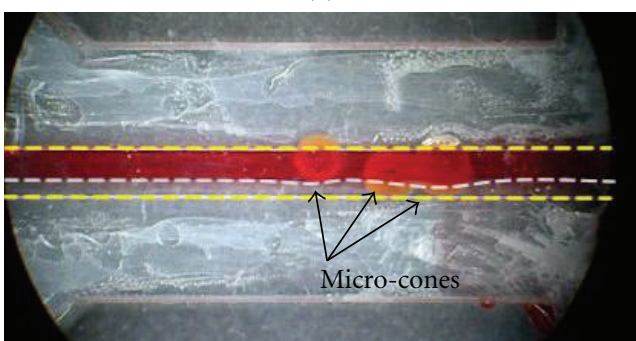

(d)

FIgURE 13: The mixing test of microchannel. (a) Deionized water in the channel without micro-cone. (b) Deionized water in the channel with three micro-cones. (c) Glycerin in the channel without micro-cone. (d) Glycerin in the channel with three micro-cones. The flow direction is from the left to the right.

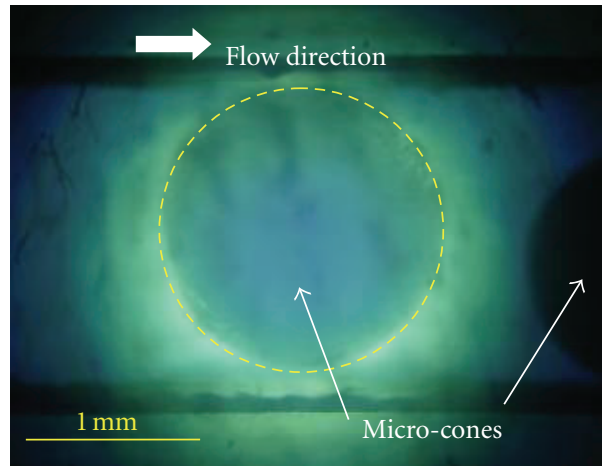

(a)

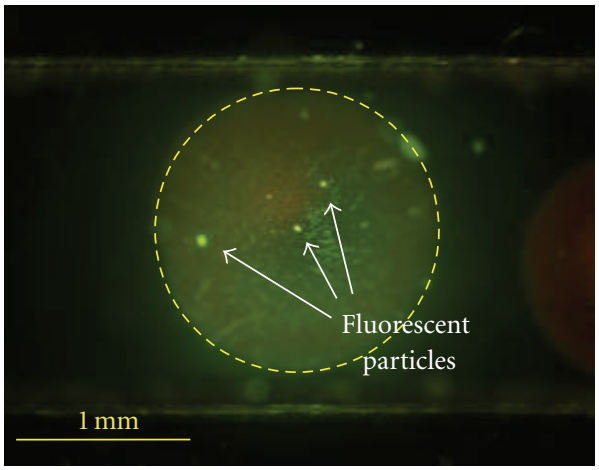

(b)

FIgURE 14: The magnetism test indicates that the micro-cone has magnetic polarity. (a) Top view of the micro-cone observed under fluorescence microscope without blue light filter. (b) The fluorescent particles captured by the micro-cone, observed under fluorescence microscope with blue light filter.

of the micro-cones test. The $50 \mu \mathrm{L}$ of streptavidin-coated magnetic particles (Dynabeads M-270 streptavidin, Invitrogen, $2.8 \mu \mathrm{m}$ in diameter, $10 \mathrm{mg} / \mathrm{mL}$ ) are mixed with $1 \mathrm{~mL}$ of biotin which were labeled with fluorescence (Biotin-4fluorescein, Molecular Probes, Invitrogen) at $20 \mu \mathrm{g} / \mathrm{mL}$ in concentration for $30 \mathrm{~min}$. In the beginning, the deionized water is injected in and full of the microchannel to expel air. The reagent is continued to flow into the microchannel at $5 \mathrm{~mL} / \mathrm{h}$ flow rate. Then, the deionized water is used to wash the reagent out of the channel. The chip is mounted on a fluorescence microscope (BX51, Olympus, Japan), and the images are taken by CCD camera. Figure 14(a) shows the microchannel full of reagent, which is observed under the microscope without the blue light filter. Figure 14(b) is the same location of the microchannel as Figure 14(a) but is observed by microscope with blue light filter. The fluorescent particles captured on the micro-cone are observed. In order to confirm that the particles are truly attracted on the microcone, the deionized water is used to wash the channel again. The particles still remain on the micro-cone after the wash, which proves that the iron(III)-oxide nanoparticle/PDMS cone has the magnetism and could be used in capturing magnetic particles.

\section{Conclusion}

An approach to fabricate the 3D iron(III)-oxide nanoparticle/PDMS cone in a microchannel has been demonstrated. The $6.38 \%$ weight ratio of the nanoparticles in the PDMS was used. An automatic dispenser was used to align the composite cone in the microchannel and to control the diameter of the composite droplet. The dispensing pressure is $0.25 \mathrm{MPa}$, and the dispensing time is $5 \mathrm{~s}$. A permanent magnet connected to the vertical manipulation stage was 
used to attract the droplet to form the cone shape. The distance between the magnet and the PMMA substrate is $1.5 \mathrm{~mm}$. The cone is cured by heating at $70^{\circ} \mathrm{C}$ for $30 \mathrm{mins}$.

The micro-cone has been successfully fabricated in the microchannel. The height of the cone is $300 \mu \mathrm{m}$, and the deviated location of the cone from the central line of the microchannel is $0.3 \mathrm{~mm}$. The cone has the magnetism due to the arrangement of the nanoparticles and can enhance the mixing efficiency. The fabricated cone in the microchannel could be applied in lab on a chip in the future.

\section{Conflict of Interests}

The authors do not have any conflict of interests in this present work.

\section{Acknowledgment}

This work has been sponsored by the National Science Council, Taiwan under the Contract no.: NSC 99-2221-E002-193-MY3.

\section{References}

[1] A. D. Stroock, S. K. W. Dertinger, A. Ajdari, I. Mezić, H. A. Stone, and G. M. Whitesides, "Chaotic mixer for microchannels," Science, vol. 295, no. 5555, pp. 647-651, 2002.

[2] Y. Z. Liu, B. J. Kim, and H. J. Sung, "Two-fluid mixing in a microchannel," International Journal of Heat and Fluid Flow, vol. 25, no. 6, pp. 986-995, 2004.

[3] H. J. Sheen, C. J. Hsu, T. H. Wu, H. C. Chu, C. C. Chang, and U. Lei, "Experimental study of flow characteristics and mixing performance in a PZT self-pumping micromixer," Sensors and Actuators A, vol. 139, no. 1-2, pp. 237-244, 2007.

[4] A. P. Sudarsan and V. M. Ugaz, "Fluid mixing in planar spiral microchannels," Lab on a Chip, vol. 6, no. 1, pp. 74-82, 2006.

[5] M. Zhang, J. Wu, L. Wang, K. Xiao, and W. Wen, "A simple method for fabricating multi-layer PDMS structures for 3D microfluidic chips," Lab on a Chip, vol. 10, no. 9, pp. 11991203, 2010.

[6] S. G. Grancharov, H. Zeng, S. Sun et al., "Bio-functionalization of monodisperse magnetic nanoparticles and their use as biomolecular labels in a magnetic tunnel junction based sensor," The Journal of Physical Chemistry B, vol. 109, no. 26, pp. 13030-13035, 2005.

[7] M. A. M. Gijs, "Magnetic bead handling on-chip: new opportunities for analytical applications," Microfluidics and Nanofluidics, vol. 1, no. 1, pp. 22-40, 2004.

[8] A. Sandhu, H. Handa, and M. Abe, "Synthesis and applications of magnetic nanoparticles for biorecognition and point of care medical diagnostics," Nanotechnology, vol. 21, no. 44, Article ID 442001, 2010.

[9] A. R. Kose and H. Koser, "Ferrofluid mediated nanocytometry," Lab on a Chip, vol. 12, no. 1, pp. 190-196, 2012.

[10] N. Pamme and C. Wilhelm, "Continuous sorting of magnetic cells via on-chip free-flow magnetophoresis," Lab on a Chip, vol. 6, no. 8, pp. 974-980, 2006.

[11] S. A. Peyman, A. Iles, and N. Pamme, "Mobile magnetic particles as solid-supports for rapid surface-based bioanalysis in continuous flow," Lab on a Chip, vol. 9, no. 21, pp. 3110 3117, 2009.
[12] T. Zhu, F. Marrero, and L. Mao, "Continuous separation of non-magnetic particles inside ferrofluids," Microfluidics and Nanofluidics, vol. 9, no. 4-5, pp. 1003-1009, 2010.

[13] K. S. Kim and J. K. Park, "Magnetic force-based multiplexed immunoassay using superparamagnetic nanoparticles in microfluidic channel," Lab on a Chip, vol. 5, no. 6, pp. 657664, 2005.

[14] S. S. Shevkoplyas, A. C. Siegel, R. M. Westervelt, M. G. Prentiss, and G. M. Whitesides, "The force acting on a superparamagnetic bead due to an applied magnetic field," Lab on a Chip, vol. 7, no. 10, pp. 1294-1302, 2007.

[15] C. Pascal, J. L. Pascal, F. Favier, M. L. E. Moubtassim, and C. Payen, "Electrochemical synthesis for the control of $\gamma$ - $\mathrm{Fe}_{2} \mathrm{O}_{3}$ nanoparticle size, morphology, microstructure, and magnetic behavior," Chemistry of Materials, vol. 11, no. 1, pp. 141-147, 1999.

[16] W. H. Binder, H. Weinstabl, and R. Sachsenhofer, "Superparamagnetic ironoxide nanoparticles via ligand exchange reactions: organic 1,2-diols as versatile building blocks for surface engineering," Journal of Nanomaterials, vol. 2008, Article ID 383020, 10 pages, 2008.

[17] C. Zhang and X. Xie, "Controllable assembly of hydrophobic superparamagnetic iron oxide nanoparticle with mPEG-PLA copolymer and its effect on MR transverse relaxation rate," Journal of Nanomaterials, vol. 2011, Article ID 152524, 7 pages, 2011.

[18] G. Filipcsei and M. Zrínyi, "Magnetodeformation effects and the swelling of ferrogels in a uniform magnetic field," Journal of Physics Condensed Matter, vol. 22, no. 27, Article ID 276001, 2010. 

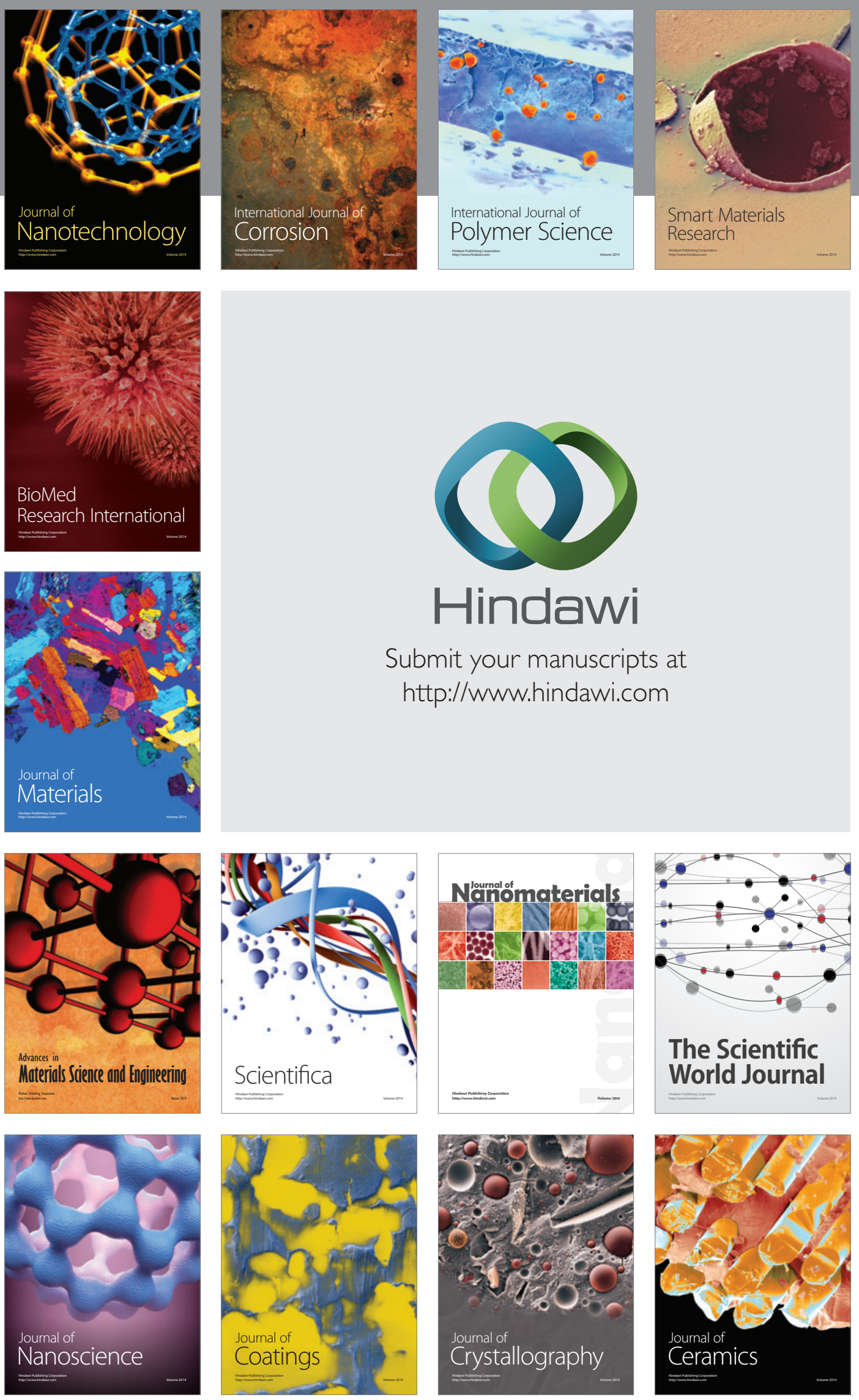

The Scientific World Journal

Submit your manuscripts at

http://www.hindawi.com

\section{World Journal}

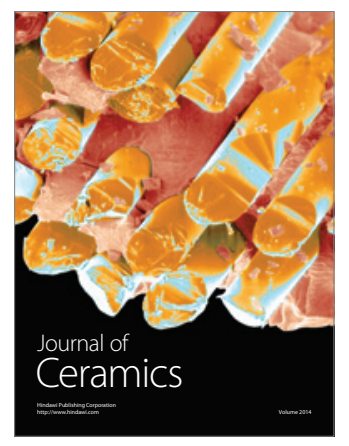

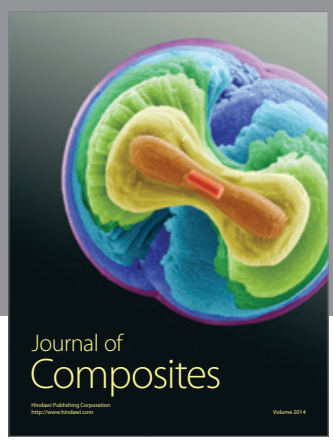
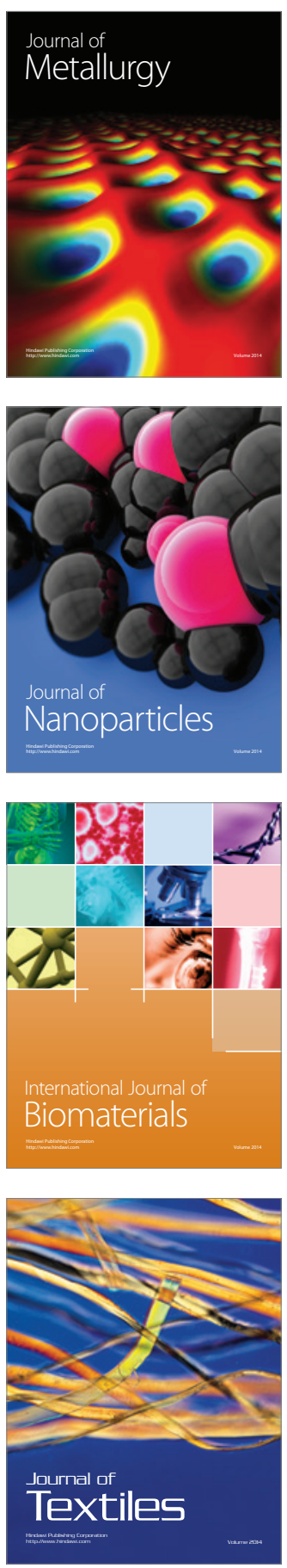